\title{
Anabases
}

Seduction and Power: the Representation of Antiquity in the Visual and Performing Arts ( ${ }^{\text {nd }}$ Imagines Project Conference. University of Bristol, UK, Bristol $22^{\text {nd }}-25^{\text {th }}$ of September 2010 )

Martina Treu et Maddalena Giovannelli

\section{(2) OpenEdition}

Journals

Édition électronique

URL : http://journals.openedition.org/anabases/2840

DOI : 10.4000 /anabases.2840

ISSN : 2256-9421

Éditeur

E.R.A.S.M.E.

\section{Édition imprimée}

Date de publication : 1 octobre 2011

Pagination : 209-213

ISSN : $1774-4296$

\section{Référence électronique}

Martina Treu et Maddalena Giovannelli, « Seduction and Power: the Representation of Antiquity in the Visual and Performing Arts ( $2^{\text {nd }}$ Imagines Project Conference. University of Bristol, uk, Bristol $22^{\text {nd }}-25^{\text {th }}$ of September 2010) », Anabases [En ligne], 14 | 2011, mis en ligne le 01 octobre 2014, consulté le 20 octobre 2019. URL : http://journals.openedition.org/anabases/2840 ; DOI : 10.4000/anabases.2840 
Anabases 14 (2011), p. 209-213.

\section{Seduction and Power: the \\ Representation of Antiquity \\ in the Visual and Performing Arts \\ $\left(2^{\text {nd }}\right.$ Imagines Project Conference. \\ University of Bristol, UK, Bristol \\ $22^{\text {nd }}-25^{\text {th }}$ of September 2010)}

Martina Treu,

Maddalena Giovannelli

L'UnIVERSITÀ dI BRISTOL OSPITA QUEST'ANNO UN CONVEGNO genuinamente internazionale per le molte nazionalità rappresentate da relatori e pubblico: un folto gruppo di spagnoli, inglesi, tedeschi, portoghesi, statunitensi e sudamericani, più un nutrito drappello di italiani (tra cui chi scrive e Maddalena Giovannelli, Andrea Capra, Erika Notti). Decisamente internazionale, del resto, è anche il ventaglio di relazioni e argomenti trattati, come si conviene a un progetto multilingue e interdisciplinare come quello in questione: denominato Imagines - Antiquity in the Visual and Performing Arts (Imagines-project.org) e avviato anni fa da studiosi di diversi Paesi (allora gravitanti attorno a Heidelberg e ora dislocati nelle università europee che partecipano al progetto, come La Rioja, Bristol e Lampeter). Gli italiani Irene Berti e Filippo Carlà, le spagnole Pepa Castillo e Marta García Morcillo, la tedesca Silke Knippschild proseguono con questo appuntamento a Bristol un percorso pluriennale che ha già prodotto in tre anni convegni e pubblicazioni.

L'ambizioso proposito è studiare in maniera innovativa e multidisciplinare le «immagini» dell'antichità - come recita il sottotitolo di Imagines - «nelle arti visive e performative»: da quelle tradizionali e via via assurte a discipline autonome - pittura, scultura, architettura, teatro, opera, fotografia, cinema - alle forme artistiche più recenti ed eterogenee come l'illustrazione pubblicitaria, il fumetto, i film d'animazione. 
Questi ultimi campi d'indagine, com'è noto, stentano ad acquisire lo status di oggetto di studio autonomo in molti ambienti accademici e a meritare spazio nella critica specialistica: devono infatti fare i conti con pregiudizi duri a morire - contro la cosiddetta letteratura "di consumo», «divulgativa» o "per ragazzi» - soprattutto da parte della «vecchia guardia» degli antichisti. Le più giovani leve come il gruppo Imagines, invece, si confrontano con simili ambiti di studio e ricerca senza preclusioni, con occhi nuovi, nella convinzione condivisa che tutti i mezzi d'espressione artistica attingano a un formidabile patrimonio di immagini costituito nel tempo, lo incrementino a loro volta e ne favoriscano la trasformazione e la diffusione, contribuiscano in modo determinante a formare l'immaginario collettivo e a raggiungere un pubblico sempre più vasto. Questi presupposti sono alla base non solo del presente convegno, ma dell'intero progetto Imagines e di altri avviati contemporaneamente in diversi paesi europei (tra cui alcune università italiane impegnate nel progetto DigITAL, in fase di studio, presentato al Convegno di Bristol da Erika Notti).

Simili programmi interdisciplinari sono tra $\mathrm{i}$ più promettenti e dal maggior potenziale, a nostro avviso, per le possibilità che offrono alla ricerca e alla didattica: anziché limitarsi a studi iconografici parziali, o a repertori singoli e isolati, ci si propone di incrementare la circolazione di informazioni, favorire la condivisione e l'indicizzazione di archivi digitali iconografici e multimediali, ampliare gli orizzonti della ricerca e contrastare i rischi di un progressivo isolamento e ripiegamento su se stesse delle discipline antiche e delle scienze umane. Le metodologie e gli oggetti di studio promossi finora dal gruppo Imagines si prestano bene ad essere utilizzati sia nelle indagini sul campo sia nell'insegnamento delle discipline antiche in scuole e università. Proprio a questo tema, la didattica, era dedicata una sezione del primo volume del gruppo, pubblicato due anni fa a seguito del primo convegno di Imagines (2007), all'università La Rioja (Imagines. La Antigüedad en las Artes Escénicas y Visuales, Universidad de La Rioja, Logroño, 2008: si veda per il programma con abstract degli interventi <http:// www.unirioja.es/cu/mjcasti/en/index.html/>).

Da quel primo convegno introduttivo - ricco di contributi eterogenei per soggetto, lingua, epoca e genere - si sono diramati negli anni diversi filoni di indagine, condotti in parallelo dai vari membri del gruppo e confluiti nel convegno di Bristol. In particolare il cinema è stato il filo conduttore di un volume curato da Irene Berti e Marta García Morcillo (Hellas on Screen. Cinematic Receptions of Ancient History, Literature and Myth, Stuttgart, Franz Steiner Verlag, 2008) e mantiene un ruolo di assoluto rilievo nell'appuntamento di quest'anno, grazie anche alle due curatrici: Berti e García Morcillo, oltre ai due interventi personali (dedicati rispettivamente a Circe e Marco Antonio) nella giornata inaugurale del 22 settembre introducono e proiettano preziosi frammenti superstiti di alcuni film muti delle origini (Nerone, 1909; Marcantonio e Cleopatra, 1913; Teodora, 1921; Salomé, 1923), accompagnati da musiche per pianoforte e violino appositamente composte ed eseguite live da due studenti.

Altri interventi illustrano con pellicole più o meno note il tema del convegno - seduzione e potere - declinato variamente in film per la tv come Odissea (1968) 
e Eneide (1971) di Franco Rossi (analizzati da Martin Winkler, uno dei massimi esperti di cinema e mondo antico), o nei vari esempi di molte nazionalità - tratti pressoché da ogni filone del cinema, dallo storico-mitologico al peplum, dal comico all'erotico - che hanno per protagonisti personaggi storici, pseudostorici o di finzione (Alessandro Magno, Caligola, Spartaco) e soprattutto «donne di potere» - regine e imperatrici - come Teodora, Cleopatra o Agrippina. Simili figure si ritrovano anche in altri contributi che spaziano dalle arti figurative a quelle sceniche, dal teatro all'opera, dall'animazione al fumetto. Riguardo a quest' ultimo aspetto va sottolineato che l'ospite d'onore del convegno è stato Eric Shanower, l'autore della pluripremiata saga a fumetti Age of Bronze (monumentale trasposizione dell'intera saga troiana, pubblicata in più volumi in Usa dal 1998 e in Italia dal 2006: L'età del bronzo, Città di Castello, Free Books).

A suggellare l'avvenuto superamento dei tradizionali confini tra arti «maggiori» e «minori», ma anche tra accademia e "cultura popolare», l'artista statunitense espone le sue tavole originali in una mostra personale alla Bristol Gallery - visitabile nei giorni del convegno - e tiene nello stesso luogo una relazione finale sulle storie di seduzione e potere che animano l'epica omerica e ispirano il suo lavoro. Si chiude così degnamente un convegno dal programma vario eppure coeso, ricco di testimonianze di prima mano e di addetti ai lavori, non a caso inaugurato da una performance multimediale. La vocazione interdisciplinare del gruppo esce certamente rafforzata da questo appuntamento, che dovrebbe essere seguito da una pubblicazione - e auspicabilmente una specifica collana "Imagines» - e da un nuovo convegno previsto per il 2013: dopo la trasferta oltre Manica si vorrebbe tornare «sul continente», in un Paese ancora da definire. Perché non l'Italia?

Martina TREU
Libera Università di Lingue e Comunicazione
IULM University
martinatreu@libero.it

Seduzione e potere. Ma anche: il potere della seduzione o la seduzione del potere. Questi i due fili rossi - da sempre intrecciati inestricabilmente l'uno all'altro - proposti dagli organizzatori (meglio: dalle organizzatrici) per circoscrivere il campo di indagine e rendere più agevole il confronto tra i relatori.

A trionfare sono naturalmente le protagoniste femminili: Cleopatra e Teodora, in primis, donne fatali per eccellenza e ritratte dagli oratori per lo più attraverso la filmografia. E se Francisco Pina Polo segue il filo della regina d'Egitto, Marta García 
Morcillo mostra l'altro lato della medaglia: quello di Antonio, sedotto e condannato per sempre a scomparire, sovrastato dal carisma dell'ultima terribile amata. Eroine tragiche decise e implacabili, vere e proprie bad girls, sono oggetto dell'intervento di Martina Treu: Clitemnestra e Medea vengono mostrate in alcuni allestimenti teatrali (la prima è un'affascinante ed energica regina nell'impeccabile allestimento di Peter Stein), mentre le Erinni (o Furie) ispirano diverse serie televisive e a fumetti.

Tra le proverbiali donne potenti e senza scrupoli, non può mancare la romana Agrippina: se ne occupa Mary H. Mc Hugh, che, passando per Mio figlio Nerone di Alberto Sordi, ripercorre i luoghi cinematografici insospettabili, a una prima lettura, dove è invece possibile rintracciare il ritorno e la declinazione di una simile tipologia di madre. Crudele e irresistibile incantatrice appare anche Circe nell'arte del rinascimento ritratta non di rado accanto ad animali dall'aspetto angosciosamente antropomorfo (relazione di Irene Berti). Regine determinate, incantevoli, e dotate di una straordinaria forza d'animo sono le tre donne immortalate dalla regia di Franco Rossi: Martin Winkler ha mostrato - proiettando ampi spezzoni di film divenuti per la maggior parte irreperibili - analogie e punti di contatto tra una Elena dai tratti orientali, una Didone che si muove con determinazione tra le piane dell'Afghanistan (qui il set del film), e una Penelope portata a nuova vita da una sorprendente Irene Papas.

Modello di donna positivo, finalmente, è invece la giovane Ifigenia: ma Maite Clavo la mostra - in un allestimento firmato da Jordi Coca - sedotta dal suo ruolo di salvatrice della patria al punto di diventare un fantoccio tra le mani degli altri personaggi. Non mancano anche le figure maschili, pur in schiacciante minoranza: primo tra tutti l'Alessandro Magno dell'hollywoodiano Alexander. Lloyd Llewellyn-Jones consulente del team di Oliver Stone oltre che docente alla University of Edinburgh - ha analizzato con acume l'incoerenza di alcune scelte registiche. In contrasto con la volontà di lanciare l'opera come un fedele film storico, la rappresentazione dell'oriente sembra risentire di alcuni pregiudizi e stereotipi ben radicati a Hollywood.

La figura di Caligola, tra pazzia e perversione, è invece seguita con scrupolo da Martin Lindner, dalle fonti romane fino alle propaggini Web, dove il temibile imperatore romano viene utilizzato in giochi di società o per pubblicizzare biancheria intima. E ancora il ribelle per eccellenza, Spartaco, è il protagonista di un film dell'italiano Riccardo Freda: a parlarne è Oscar Lapeña, che mette in luce le profonde differenze tra questa edizione e la successiva e più fortunata di Stanley Kubrick.

Uomini e donne di potere, dunque, ma non solo. Charlotte Ribeyrol si occupa di una labile linea di confine, mostrando la ricorrenza del motivo dell'ermafrodito nella pittura degli esteti inglesi. Nicoletta Momigliano tratta invece una seduzione in qualche modo anomala: quella della Creta minoica, capace di affascinare Isadora Duncan al punto da indurla a una estemporanea - e non da tutti gradita - performance di danza sugli scavi di Cnosso.

Questi sono solo alcuni dei fili rossi (ed è stata notata più volte, durante i lavori, la ricorrenza di questo colore) di seduzione e potere emersi nel corso del convegno. Nonostante il vastissimo campo di indagine e la molteplicità ed eterogeneità degli 
argomenti, non sono mancati i cortocircuiti: la filmografia di Pasolini per esempio seppure mai scelta dai relatori come esplicito campo di indagine - è stata un costante punto di confronto e di riferimento nelle discussioni. A trionfare, nei contributi e nel dibattito, è stato senz'altro il cinema italiano (in una sorta di contrappasso dell' edizione 2010 del festival di Venezia): citato e richiamato dai relatori stranieri con una tale competenza da lasciare senza parole i presenti madrelingua.

Maddalena GiovanNelLI

Università Statale di Milano

maddalena.giovannelli@stratagemmi.it 